\title{
Dual-functioning transcription factors in the developmental gene network of Drosophila melanogaster
}

\author{
Denis C Bauer*, Fabian A Buske and Timothy L Bailey
}

\begin{abstract}
Background: Quantitative models for transcriptional regulation have shown great promise for advancing our understanding of the biological mechanisms underlying gene regulation. However, all of the models to date assume a transcription factor (TF) to have either activating or repressing function towards all the genes it is regulating.

Results: In this paper we demonstrate, on the example of the developmental gene network in D. melanogaster, that the data-fit can be improved by up to $40 \%$ if the model is allowing certain TFs to have dual function, that is, acting as activator for some genes and as repressor for others. We demonstrate that the improvement is not due to additional flexibility in the model but rather derived from the data itself. We also found no evidence for the involvement of other known site-specific TFs in regulating this network. Finally, we propose SUMOylation as a candidate biological mechanism allowing TFs to switch their role when a small ubiquitin-like modifier (SUMO) is covalently attached to the TF. We strengthen this hypothesis by demonstrating that the TFs predicted to have dual function also contain the known SUMO consensus motif, while TFs predicted to have only one role lack this motif.
\end{abstract}

Conclusions: We argue that a SUMOylation-dependent mechanism allowing TFs to have dual function represents a promising area for further research and might be another step towards uncovering the biological mechanisms underlying transcriptional regulation.

\section{Background}

Site-specific transcription factors (TFs) that bind to the regulatory regions surrounding the target gene--so-called cis-regulatory-modules (CRMs)--are known to interact with the basal transcription complex to initiate transcription [1]. TFs can increase transcription by functioning as activators, or reduce transcription as repressors, respectively. The frequency and duration of the binding events is influenced by the concentration of the TF proteins, the binding affinities and location of the transcription factor binding sites (TFBSs) in the CRM, and the properties of the TFs themselves (e.g. effectiveness, competitive interaction with other TFs).

Modelling these binding events to quantitatively predict the resulting transcriptional output of the target gene has become increasingly successful [2-6]. The approaches

\footnotetext{
* Correspondence: d.bauer@uq.edu.au

1 Institute for Molecular Bioscience, The University of Queensland, Brisbane, Qld. 4072 Australia

Full list of author information is available at the end of the article
}

model interaction of TFs and DNA using thermodynamic equations and predict the transcriptional response of the target gene as mediated by these interactions. A training algorithm is used to minimize the difference between the observed and predicted transcriptional response by adjusting the model parameters.

In previous research, thermodynamic models have been trained and tested on only one CRM [3,7]. Because the flexibility of the model is unlikely to be constrained sufficiently by the small amount of data from fitting only one CRM, it is possible that these models over-fit their input data, which would render them useless for the prediction of transcriptional output of other genes [6]. Indeed, in an earlier paper we showed that a large number of different model settings were able to produce nearly identical output, which supports the over-fitting hypothesis [8]. Gertz et al. [6] showed that the model predictions are more robust when models are trained on multiple synthetically generated CRMs. Hence, training on the regulatory sequence of multiple genes regulated by 
the same TFs should increase the quality of the generated model. Aiming at increasing the confidence in the trained thermodynamic model, Segal et al. [5] trained one model to fit the expression data of 44 developmental CRMs (20 genes) in D. melanogaster. Their model was able to fit about one third of the 44 CRMs with a correlation coefficient $(\mathrm{CC}$ ) larger than 0.85 (where $C C=1$ is perfect correlation and $C C=0$ means no correlation). For the majority of the CRMs, however, the model fails to produce as good a fit, and the $\mathrm{CC}$ values are below 0.5 for a third of the data. They argue that the failure of their model can be attributed to missing higher-order interaction rules (like positive synergism) or missing input factors (especially activators).

An alternative explanation, however, is that not all 44 CRMs are regulated in the same way. Though all CRMs seem to be regulated by the same eight TFs, the role (repressor or activator) that the individual TFs have in the regulation of some CRMs might differ. Several experimental studies point to such "context-dependent" regulation for CRMs of developmental genes in D. melanogaster $[9,10]$. For example, Hunchback $(\mathrm{Hb})$ is involved in regulating the even-skipped gene (eve), which is expressed in seven stripes along the body of the $D$. melanogaster embryo. It has been postulated that $\mathrm{Hb}$ takes the role of an activator in the CRM responsible for the expression of the second stripe, while being a repressor in the CRM regulating the third stripe [11].

To date, it is unclear what biological mechanism in $D$. melanogaster might explain how a TF switches from activator to repressor or vice versa (see Reinitz et al. [12] for a review). One hypothesis is that the TF itself does not switch its function, but that the altered outcome is an emergent property of the complex interactions of the system. For example, if a strong and a weak activator share a similar DNA binding profile, at high concentrations the weak activator can out-compete the strong activator and ultimately reduce transcription despite its role as activator [10]. However, this type of interaction should be fully captured by current thermodynamic models. Another hypothesis is the concentration-dependent switch, which proposes that a TF that is an activator at low concentrations can function as repressor at high concentrations (e.g. due to excessive aggregation) [13]. Yet another hypothesis is cooperativity, where the complex of two different TFs exert the opposite function as its individual components [10]. However, a protein-protein binding experiment of TFs and colocalization studies of the TFBSs failed to support this hypothesis $[9,14,15]$.

In this article we suggest an alternative mechanism by which the developmental TFs in D. melanogaster can switch their regulatory roles. It has been shown that SUMOylation of individual TFs can lead to a loss or reversal of the regulatory function [16,17]. SUMOylation is a post-translational modification that attaches a small ubiquitin-like modifier (SUMO) covalently to a target protein [18].

The aims of this paper are twofold. Firstly, we investigate the validity of a SUMOylation-driven switching mechanism for the eight regulatory TFs in D. melanogaster. Secondly, we identify the role for each of the eight regulatory TFs that best describe the developmental gene network in $D$. melanogaster. To achieve this, we investigate the evidence for one or more TFs to have dual function, that is, function as activator for some CRMs and as repressor for others.

\section{Results and Discussion}

\section{Analyzing the issues with state-of-the-art models}

We first establish the ability of existing thermodynamic models of expression (Segal model [5] and Reinitz model [2]) to fit the Drosophila gap-gene expression data when only one role per TF is assumed. In order to measure the performance of a model, we record the correlation coefficient (CC) achieved when fitting all 44 developmental CRMs simultaneously. We assign Bicoid (Bcd), Caudal (Cad) and Torso-Response-Element (TorRE) as activator and Hunchback (Hb), Giant (Gt), Knirps (Kni), Krüppel $(\mathrm{Kr})$ and Tailless (Tll) as repressor [5]. We herein refer to this particular role assignment as literature configuration. Simulated annealing (SA) is used to optimize the Reinitz model on the 44 CRMs and the accuracy is calculated from the resulting model. The accuracy of the Segal model on the same data is taken from predictions provided by Segal et al. [5].

Table 1 shows that neither the Segal model nor the Reinitz model are able fit all of the 44 CRMs simultaneously, with neither model achieving a CC of more than 0.6. The CC of the Segal model is more than twice as high as the one achieved by the Reinitz model. However, as indicated in the table, the Segal model has also far more free parameters. In particular, the Segal model learns the PWM parameters from the data. Since there has not been a detailed study examining the influence of the different model components on the ability to fit the data, one can only speculate to what degree the higher accuracy of the

Table 1: Ability of the Segal model and Reinitz model to fit the Segal single-time data.

\begin{tabular}{lcc}
\hline $\begin{array}{l}\text { model } \\
\text { type }\end{array}$ & $\begin{array}{c}\text { number of free } \\
\text { parameters }\end{array}$ & $\begin{array}{c}\text { mean simultaneously CC } \\
\text { (SE) }\end{array}$ \\
\hline Segal & 344 & $0.59(0.009)$ \\
Reinitz & 18 & $0.27(0.008)$ \\
\hline
\end{tabular}

The second column shows the number of free parameters used in the models. The third column shows the average CC over all 44 developmental CRMs. The standard error is given in parentheses. 
Segal model results from the large number of additional free parameters. It should be kept in mind that internal flexibility can lead to good fitting-accuracy despite wrong biological assumptions also known as "over-fitting" [8]. Note, as shown in the Additional file 1 Section 5 using these PWMs learned by the Segal model also improves the accuracy of the Reinitz model in fitting the 44 CRMs compared to the standard PWMs used in previous research [7]. Because we believe that the Reinitz model may be less prone to over-fitting when trained on small data sets, and because it is far faster to train, we use it in the subsequent experiments.

\section{Determining the regulatory role for each TF}

Our next objective is to determine which of the $2^{|\mathrm{TFs}|}=$ 256 possible configurations of how to assign the roles-activator or repressor--to each of the eight TFs fits the available data best. We consider an upper bound on accuracy by allowing the roles of all TFs to (potentially) be different for each CRM. A more reasonable upper bound would be given by allowing CRMs to choose from only a small number of configurations. Preferences for a certain role by a TF from each of these scenarios can suggest its true role for a given CRM. We also explore a sensitivitybased approach that measures the effect of changing the role of an individual TF.

There are 44 CRMs and 256 possible TF role configurations. We would like to consider all possible assignments of configurations to CRMs, and train models simultaneously using all CRMs. However, due to the huge number of possible assignments, this is technically infeasible. Instead, we train the Reinitz model using each CRM-configuration pair independently, and record the correlation between the observed CRM output and the model. This results in a $44 \times 256$ matrix of CC values, one for each CRM-configuration pair. We use this matrix in our three upper-bound and sensitivity analyses as described in the following paragraphs. We expect models trained this way to be heavily over-fitted and the CC to be overly optimistic. However, as described in Methods Sec. 0.4, by focusing on the strongest signals in the data, we are only extracting candidate role assignments for further testing in Sec. 0.1, where we train on all 44 CRMs again. We do not use the accuracy estimates otherwise.

We first establish an upper bound on fitting accuracy by finding the best possible TF role configuration for each CRM independently. We do this using what we call the SMALLEST-OPTIMAL method, $U$, which identifies the TF role configuration(s) with best CC for each CRM individually and takes the union. The the smallest subset containing at least one optimal configuration for each CRM has size 17, with an average CC of 0.71 (standard error 0.006). Since the average CC is calculated over the set of optimal configurations with an individually trained model for each CRM, it represents the upper bound regarding the accuracy that can be achieved with freely altering the TF roles between CRMs (Figure 1, dashed line). We next look for a small set of configurations that most accurately fit the data. We are able to find optimum sets (in terms of total CC) with up to 4 configurations using an algorithm we call the best-n method, $\beta$. This method searches for the set of $n$ configurations such that the total CC is minimum when each CRM is assigned one of the $n$ configurations and trained independently from the other CRMs. As seen in Figure 1, the single best role assignment $(n=1)$ identified by the method already reaches $76 \%$ of the upper bound (average CC $=0.54$, 0.007 standard error). By comparison, the literature configuration of TF roles achieves far lower accuracy (average $C C=0.45, S E=0.009$ ). These two configurations differ only in the role of $\mathrm{Kr}$, which is a repressor in the literature configuration. Also shown in Figure 1, the accuracy converges to the upper bound with the number of additional configurations allowed. We use four configurations in the subsequent parts of the paper, which achieves an average CC of 0.67 (0.006), already $94 \%$ of the best CC achievable by the model on this data (upper bound).

Finally, the SENSITIVITY method, $\Delta$, determines the role for each TF individually by identifying which change in role causes the largest change in accuracy between some

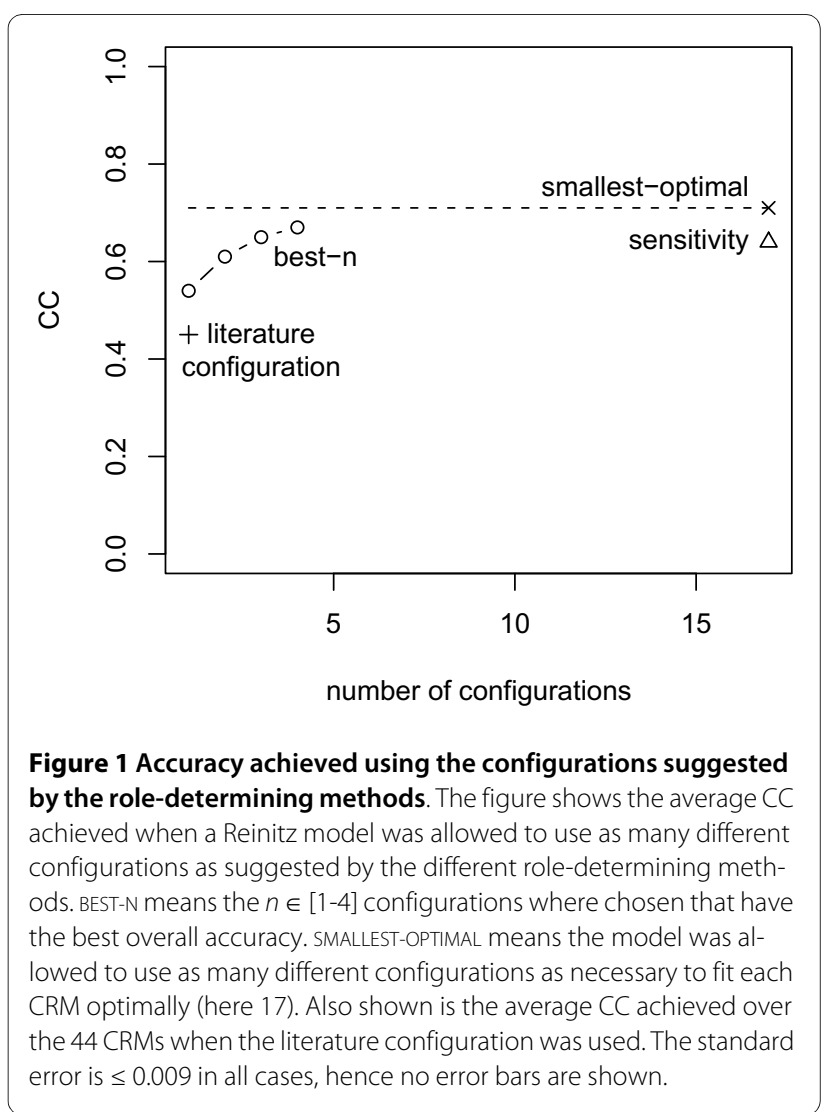


pair of configurations. This is done independently for each CRM. This method results in 17 configurations (note, these 17 configurations are not identical with the 17 obtained by the smallest-optimal method). The average accuracy achieved by the optimal configurations is $C C=0.64(S E=0.006)$, slightly below the upper bound (Figure 1). For ten out of the $44 \mathrm{CRMs}$ all three methods predict the same best configuration (see Additional file 1 Section 6. For the other $34 \mathrm{CRMs}$, the configuration predicted by at least one of the methods disagrees with those predicted by the other two. The agreement between the three methods is demonstrated for six CRMs in Table 2 (see Additional file 1 Section 6 for the full table).

The disagreement between the methods calls for the construction of a voting ensemble. We aim to integrate the different properties of best accuracy $(\cup)$, smallest number of different configurations $(\beta)$ and strongest signal $(\Delta)$ in an ensemble by combining the three methods using majority vote. Table 2 shows the role assignment over all CRMs as determined by this ensemble (Table 2, last row). To define a single role for each TF we first combine for each method individually the evidence for all CRMs and then build the majority vote from them. We combine the evidence by defining a TF to be an activator or repressor if for more than $\frac{2}{3}$ of the CRMs the TF was favoured as activator and repressor, respectively (see
Methods Sec. 0.4.2). If for fewer than $\frac{2}{3}$ of the CRMs the function agreed, we define the TF to switch roles. Based on this approach we propose that Cad is consistently an activator, Tll and Kni are consistently repressors, and $\mathrm{Bcd}, \mathrm{Hb}, \mathrm{Gt}, \mathrm{Kr}$ and Torre function as switching or "dualfunctioning" TFs. As shown in Table 2, $\mathrm{Hb}$ and $\mathrm{Kr}$ are the TFs with the "strongest" role predictions (see Methods Sec. 0.4) and provide hence the strongest evidence for dual function. We use both TFs as candidates to address the question how the dual function impacts on the model accuracy.

\subsection{Improving the data fit using dual roles}

The above results based on fitting the Reinitz model to individual CRMs strongly suggest that TFs $\mathrm{Hb}$ and $\mathrm{Kr}$ may play different roles for different CRMs. In this section, we further explore this possibility by training models simultaneously on all 44 CRMs. We train three models, which we call HbDual, KrDual and HbKrDual, that allow one or both of $\mathrm{Hb}$ and $\mathrm{Kr}$ to be assigned a specific role (activator or repressor) for each individual CRM. For each CRM, the role of $\mathrm{Hb}, \mathrm{Kr}$ or both is determined by their roles predicted by the SENSITIVITY method (see Additional file 1 Section 3 for the list of CRMs to which $\mathrm{Hb}$ or $\mathrm{Kr}$ is attributed as activator). The roles of the other TFs are fixed at the literature configuration. As before, we use the $\mathrm{CC}$ of the fit of the model to the data as a measure of accuracy.

Table 2: Assigning TF roles for different CRMs.

\begin{tabular}{|c|c|c|c|c|c|c|c|c|}
\hline CRM & Bcd & Cad & $H b$ & TII & Gt & $K r$ & Kni & Torre \\
\hline Kr_CD1_ru & $-(\Delta)$ & $+(\cup)$ & $-(\cup)$ & $-(\beta)$ & $-(\beta)$ & + & - & NA \\
\hline eve_37ext_ru & - & + & $+(\beta)$ & + & $+(\Delta)$ & NA & - & - \\
\hline eve_stripe2 & + & $+(\Delta)$ & $-(\Delta)$ & - & - & - & - & NA \\
\hline hb_anterior_actv & + & NA & $+(\beta)$ & - & $-(\Delta)$ & - & - & NA \\
\hline kni_+1 & $+(\beta)$ & $-(\Delta)$ & $-(\beta)$ & - & + & $-(\Delta)$ & + & - \\
\hline run_stripe5 & + & + & - & - & - & - & - & $-(\beta)$ \\
\hline roles & $\mathrm{s}$ & $+(\cup)$ & $\mathrm{s}(\beta)$ & $-(\cup)$ & $\mathrm{s}$ & $\mathrm{s}(\beta)$ & $-(\Delta)$ & $\mathrm{s}(\beta)$ \\
\hline confidence & 111 & 110 & 127 & 94 & 110 & 117 & 103 & 104 \\
\hline
\end{tabular}

The first rows give the predictions for the TF roles for six of the 44 CRMs (see Additional file 1 Section 1 for the complete set of predictions). The roles for each TF (columns) are determined by majority vote of the three methods: "U" - SMALLEST-OPTIMAL, " $\beta$ " - BEST-N and " $\Delta$ " SENSITIVITY. "+" means activator, "-" repressor, respectively and "NA" indicates that no "strong" role prediction could be made for the CRM. The disagreeing method, if any, is shown in brackets. The second last row gives the overall prediction of the role of the TF: activator, repressor or switcher, "s" (see main text for method). The last row shows the number of "strong" role predictions summed over the 44 CRMs and three methods (total of 132). 
Table 3: Improvement in the ability to fit the data when dual function for $\mathrm{Hb}$ and $\mathrm{Kr}$ are allowed.

\begin{tabular}{|c|c|c|c|}
\hline model type & number of configurations & number of free parameters & mean simultaneously CC (SE) \\
\hline Reinitz & 1 & 18 & $0.27(0.008)$ \\
\hline Reinitz KrDual & 2 & 19 & $0.35(0.009)$ \\
\hline Reinitz HbDual & 2 & 19 & $0.37(0.007)$ \\
\hline Reinitz HbKrDual & 4 & 20 & $0.38(0.007)$ \\
\hline Segal & 1 & 344 & $0.59(0.009)$ \\
\hline
\end{tabular}

Table 3 shows that allowing for dual function improves the ability of the Reinitz model to fit the data substantially. Allowing $\mathrm{Kr}$ to switch its role boosts the accuracy by $30 \%$ to $C C=0.35$ compared to the Reinitz model using the literature configuration $(C C=0.27)$. An even better fit is obtained when $\mathrm{Hb}$ adopts dual function $(C C=0.37)$. Allowing both $\mathrm{Hb}$ and $\mathrm{Kr}$ to switch roles further increases the data fit, however to a lesser degree, resulting in a best accuracy of $C C=0.38$ (40\% improvement).

For each TF that is allowed to switch, one additional free parameter is added to the model, since the model must contain an "effectiveness" parameter, $\tilde{E}$, for each role of switching TFs. The complexity of the model is increased further by the fact that it is essentially clustering the CRMs into two or four classes (according to the roles of $\mathrm{Hb}$ and/or $\mathrm{Kr}$ ), and modeling each class separately. However, each of these class models shares all but at most two of its free parameters with the other class models.

The relatively small gain from $\mathrm{HbDual}$ to $\mathrm{HbKrDual}$ has two implications. Firstly, there must be a large set of CRMs that benefit from either switch. This is most likely due to TFs now developing their full effectiveness rather than compromise to limit the negative effects from wrongly assigned roles. Hence, the effect of assigning the "correct" role to a particular CRM can also prove beneficial for other CRMs. The second implication is that despite the global improvement, there are TF-specific gains observable, which can only be achieved if both TFs can serve their CRMs correctly. The accuracy of each individual CRM are provided in Additional file 1 Section 1.

Additional file 1 Section 3 shows the expression profile of the CRMs attributed to $\mathrm{Hb}$ (or $\mathrm{Kr}$ ) as activator, demon- strating that the assignment is not "trivial", that is CRMs with activation coinciding with the protein expression of $\mathrm{Hb}$ are not all assigned to the $\mathrm{Hb}$ activator set. For example, $k n i_{-}+1$ and $k r_{-} C D 2 \_r u$ have a transcriptional output at the AP position where $\mathrm{Hb}$ is expressed, yet they do not require $\mathrm{Hb}$ to be an activator.

These three effects are visualized in Figure 2. A model with literature configuration assignment achieves a CC of 0.13 for $k r \_C D 1 \_r u$, which can be improved by HbDual to 0.41 , however the peak of the prediction is clearly shifted posterior by both models. KrDual corrects this shift considerably increasing the accuracy to 0.676 , however has an extension of the activation anterior, which can only be corrected by the HbKrDual model. The latter allows for a stronger repressing $\mathrm{Hb}$ at the required position (40\% AP), achieving a CC of 0.681 . Similarly, the initial accuracy of $h b$ _anterior_actv of 0.36 can be improved by KrDual reducing the falsely predicted posterior peaks but only HbDual and HbKrDual can improve the accuracy to capture the shape with $C C=0.93$ correctly. The CRM kni_+1 is an example for which accuracy improvement from HbDual, KrDual and HbKrDual are identical. For these three models the role of either $\mathrm{Hb}$ or $\mathrm{Kr}$ or both deviated from the literature configuration. There are also accuracy gains due to indirect effects such as a more accurate parameter estimation, as shown with run_stripe5, for which the roles of $\mathrm{Hb}$ and $\mathrm{Kr}$ do not change with regard to their respective literature configuration. eve_37ext_ru is an example where only the switch of one TF increases accuracy, while (additional) switching of the other TF results in negative effects. The Reinitz model initially achieves a CC of 0.22 on eve_37ext_ru, which improves to 0.58 by $\mathrm{HbDual}$ but then decreases to 0.48 in HbKrDual. This might be due to a wrong role assignment obtained by the SENSITIVITY method for $\mathrm{Kr}$. The same reason may be responsible for a decrease in accuracy on a specific CRM for any investigated Dual model as exemplified with eve_stripe2. The question 


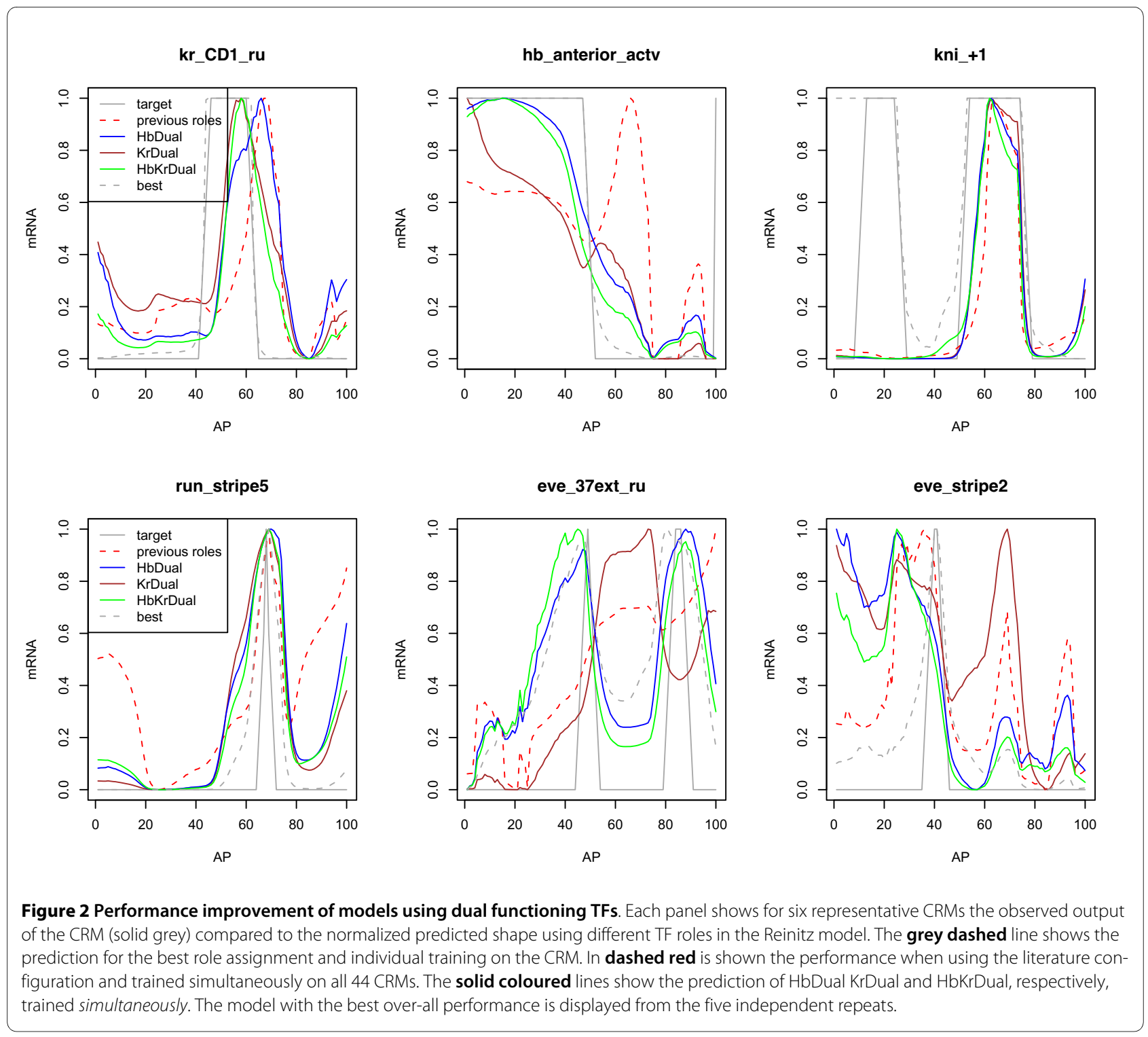

remains if other TFs than the investigated eight are involved in the regulation of developmental genes and can account for the observed behavior of $\mathrm{Hb}$ and $\mathrm{Kr}$.

Determining the involvement of other TFs in the regulation In this section we test if other regulatory TFs might be involved in the regulation of developmental genes. Since these (additional) TFs are not included during the training of the model, the proposed dual-functioning TFs might mimic or compensate for their task rather than being indeed dual-functioning in vivo.

If such a site-specific TF is missing from the set of regulators, it should have TFBSs in the regulatory regions of D. melanogaster. Searching in the sequence of all 44 developmental CRMs for enriched sites might indeed retrieve a yet unknown TF that is involved in the regulation. However, without the TF's expression profile we would not be able to make any inferences whether the TF's involvement is a better explanation for the observed regulatory differences than the dual-functioning of one of the eight regulatory TFs. Finding a motif that is enriched in one set of CRMs relative to the other set, however, would be a potential explanation for why the CRMs act differently.

In the previous section, we already identified two sets of CRMs with different regulatory mechanisms, one with $\mathrm{Hb}$ favoured as activator and the other where $\mathrm{Hb}$ is preferred a repressor. If a missing TF, rather than the dual role of known TFs, is responsible for the difference in regulation between the two CRM sets, the TFBSs of this unknown TF should be enriched in one set, while underrepresented or background distributed in the other. The enrichment should be more pronounced than when comparing to non-regulatory sequence. The same should be 
the case with the set of CRMs favouring divergent roles for Kr. We use CLOVER [19] to identify which Drosophila TFs with known binding profiles are statistically overrepresented or underrepresented in the sequences of the Hb_act set, compared to the Hb_rep set (see Methods Sec. 0.6 for more details), and we do likewise for Kr.

The enrichment $p$-values for the $\mathrm{Hb}$ and $\mathrm{Kr}$ sets, compared to all regulatory genomic regions in $D$. melanogaster, are shown in Table 4 We identify 12 out of the 76 known site-specific TFs to be over-represented in one of the four sets of CRMs. Only three are differentially enriched--that is overrepresented in the activator set and underrepresented or background distributed in the repressor set (or vice versa).

For the Hsf and Kni motifs, one set of sequences was enriched for the motif with a CLOVER[19] $p$-value (uncorrected) of 0 , which actually represents a $p$-value of "no greater than 0.001" [19]. Since 76 motifs were considered, the corresponding Bonferroni corrected $p$-value is 0.076 , so we can state that these motifs are enriched at that significance level. For these motifs, the other sets of sequences had uncorrected $p$-values greater than 0.5 , and corrected $p$-values close to 1 , so these motifs were definitely not enriched in the alternate sequence sets. In the case of the Br-Z4 motif, one set of sequences has an uncorrected $p$-value of 0.006 , which is not significant when corrected. Nonetheless, we suspect that this motif is enriched in that set of sequences, and not enriched in the other set, where the uncorrected $p$-value is 0.821 .

However, based on prior knowledge of the functions of these three candidates, none of them seems to be a missing TF for the regulation during development. Hsf does not seem to be present during gastrulation, according to staining images from http://www.flyexpress.net, and hence cannot influence the expression of the developmental genes. Br-Z4 has a binding profile similar to $\mathrm{Bcd}$ $($ Tомтом[20] p-value $=0.0091$, see Additional file $1 \mathrm{Sec}$ tion 2), which is enriched in both sets. The under-representation of $\mathrm{Br}-\mathrm{Z} 4$ is hence likely due to the higher proportion of the $\mathrm{Bcd}$-version of the motif rather than the true absence of any binding sites of Br-Z4. Finally, the under-representation of $\mathrm{Kni}$ in the $\mathrm{Kr}_{-}$act set can be explained by the observation that a large proportion of the CRMs that prefer $\mathrm{Kr}$ to be an activator produce expression that coincides with the AP position of highest Kni concentration (see Additional file 1 Section 3). Since $\mathrm{Kni}$ is consistently a repressor, its binding would inhibit activation, which makes the absence of Kni TFBSs in those CRMs favourable and explains the under-representation of Kni sites in the $\mathrm{Kr} \_$act set. It is noteworthy that

Table 4: TFs with enriched TFBSs in CRMs where $\mathrm{Hb}$ or $\mathrm{Kr}$ are activators or repressors.

\begin{tabular}{|c|c|c|c|c|}
\hline \multirow[t]{2}{*}{$T F$} & \multicolumn{2}{|c|}{$\begin{array}{c}H b \\
p \text {-value for set }\end{array}$} & \multicolumn{2}{|c|}{$\begin{array}{c}\text { Kr } \\
p \text {-value for set }\end{array}$} \\
\hline & act & rep & act & rep \\
\hline \# of CRMs & 17 & 27 & 11 & 33 \\
\hline Abd-B & 0.0 & 0.0 & 0.0 & 0.0 \\
\hline Deaf1 & & & 0.079 & 0.005 \\
\hline His2B & 0.048 & 0.0 & 0.0 & 0.058 \\
\hline $\mathrm{Hsf} *$ & 0.085 & 0.008 & 0.0 & 0.231 \\
\hline $\mathrm{Kr}$ & 0.0 & 0.0 & 0.0 & 0.0 \\
\hline $\mathrm{Bcd}$ & 0.0 & 0.0 & 0.0 & 0.0 \\
\hline $\mathrm{Br}-\mathrm{Z4}^{+}$ & & & 0.821 & 0.006 \\
\hline Cad & 0.002 & 0.0 & 0.0 & 0.0 \\
\hline $\mathrm{Hb}$ & 0.032 & 0.0 & 0.0 & 0.0 \\
\hline Kni & 0.032 & 0.01 & 0.702 & 0.0 \\
\hline TII & 0.003 & 0.0 & 0.0 & 0.0 \\
\hline Ttk & 0.02 & 0.0 & 0.003 & 0.0 \\
\hline
\end{tabular}

The first row shows the number of CRMs in each set. Each following row shows a TF out of the 76 tested for which a Clover [19] analysis resulted in a significant over- or under-representation in the sequences where $\mathrm{Hb}$ is preferred as activator (second column) or repressor (third column) or Kr preferred as activator (fourth column) or repressor (fifth column), respectively. Highlighted in bold are the cases with differential enrichment between the activator and repressor set. The TF marked with "**" is not expressed during the developmental time points $\mathrm{C} 13$ and $\mathrm{C} 14$ as determined from in-situ staining images http://www.flyexpress.net. The TF marked with "+" has a binding profile that is very similar to Bcd. Empty cells indicate that the TF was not significant for the sequence sets of $\mathrm{Hb}$. 
binding sites for Slp1, which was suggested by Andrioli et al. [21] to be involved in the regulation of some of the CRMs studied here, are neither over- nor under-represented) in any of the CRMs (data not shown). Hence, regulation by Slp1 appears not able to account for the (apparent) differing regulatory function of the other TFs.

\section{Analyzing SUMOylation as the mechanism for dual- functioning TFs}

As described in the previous section, we failed to find an enriched TF binding motif, which strengthens the hypothesis of dual-functioning TFs. In this section we explore the protein sequences of the TFs proposed to be dual-functioning to see if there exists a biological marker that discriminates them from proteins proposed to have a single role. We therefore search for short motifs $(\approx 5 a a)$ common in all protein sequences of TFs with dual function using MEME [22] for finding one occurrence per sequence, "OOPS". In order to identify motifs that generally appear in the eight regulatory sequences, we also scan the protein sequences of TFs predicted to have single roles.

The results of these scans are shown in Figure 3. As motifs 2 and 10 appear also in the protein sequence of TFs predicted to have a single role (motifs 1 and 5), they can hence be disregarded as marker. Motif 4 does not have a high information content because no sequence position was fully conserved amongst the five protein sequences, which indicates that the motif may only represent noise. This narrows the search for a marker down to two motifs: Motif 6, which is $\Psi$ K. E, where " $\Psi$ " represents a aliphatic amino acid (I, L, V) and "." represents any amino acid, and motif 8, which is $\Phi C . I$, where "Ф" represents a hydrophobic amino acid $(\mathrm{K}, \mathrm{L}, \mathrm{Y})$. While a literature search revealed no information about motif 8 , motif 6 was in fact identified as the known SUMOylation consensus motif. SUMOylation is the post-translational modification, where a SUMO protein, is covalently attached to the target protein. As a matter of fact, SUMOylation has been described to alter the transcriptional function of a TF. Additional file 1 Section 4 shows the positions of the SUMOylation consensus motifs with respect to other protein domains in the eight TFs [23].

Table 5 summarizes for each TF the roles as suggested by this research and the presence of SUMOylation consensus motifs. All of the TFs predicted to switch roles contain a SUMOylation consensus motif and all TFs predicted to have a single role lack this motif. The suggested role for $\mathrm{Cad}, \mathrm{Hb}$, Tll, Gt and $\mathrm{Kr}$ is in agreement with the literature, which used distinct methods to derive this information.

It is believed that the functional role of a TF can be mediated by the SUMO-dependent interaction with different cofactors. Valin et al. [24] validated this hypothesis for $\mathrm{Sp} 3$, a Zinc finger $\mathrm{C} 2 \mathrm{H} 2$-protein like $\mathrm{Hb}$, where SUMOylation promotes the interactions with a corepressor protein causing the complex to repress transcription, whereas a non-SUMOylated Sp3 protein promotes transcription. SUMOylation has also been shown to increase transcriptional activation [25,26]. Another related example is Ikaros, the human homologue of Krüppel (Kr), whose ability to repress is reduced when SUMOylated [27]. The loss or reduction of repressor function in concert with the competition for binding sites with a stronger repressor can result in overall activation [10]. Furthermore, Stielow et al. [28] showed that a SUMO-modified TF can silence genes by triggering the formation of local heterochromatin-like structures. Specifically, they showed that a modified $\mathrm{Sp} 3$ protein recruits chromatin remodelling proteins. It remains to be shown whether one of these mechanisms can account for the postulated functional change in the role of the TFs and whether an interaction with SUMO is both protein-concentration and CRM dependent.

\section{Conclusions}

In this study we investigated the developmental gene network of D. melanogaster. Using a thermodynamic model, we studied the effect of TFs taking opposite roles for distinct sets of CRMs. We identified five TFs with potentially dual roles from the data and investigated the two TFs that provide the strongest evidence for having dual function, Hunchback $(\mathrm{Hb})$ and Krüppel $(\mathrm{Kr})$. Our identification of these two TFs as potentially acting both as activators and repressors agrees with previously reported evidence $[9,29]$. We show that the accuracy with which our chosen thermodynamic model can be fit to existing gene expression data increases by $40 \%$ when both $\mathrm{Kr}$ and $\mathrm{Hb}$ are allowed to have dual function, and by $30 \%$ and $37 \%$ respectively when $\mathrm{Kr}$ or $\mathrm{Hb}$ are allowed to switch.

Our results do not support the previously hypothesized role of $\mathrm{Hb}$ as a repressor in the CRM regulating the third and seventh stripe of eve expression, MSE3+7 [11]. We predict that $\mathrm{Hb}$ is an activator for MSE2 and MSE3 +7 and a repressor for the remaining eve stripes. Interestingly, $M S E 2$ and MSE3+ 7 are located upstream of the eve gene, while the other MSEs are located downstream. Our findings also disagree with the concentration-dependent switching mechanism proposed by Papatsenko et al. [13], as CRMs requiring $\mathrm{Hb}$ to act as repressor do not drive peaks in expression at locations in the embryo with the highest concentration of $\mathrm{Hb}$ protein.

We also explore in silico the possibility that another known site-specific TF might be binding to the CRMs used in our study. No known TF DNA-binding motif is differentially enriched in the CRMs in which $\mathrm{Hb}$ (or $\mathrm{Kr}$ ) appears to act as an activator compared with those where it appears to act as a repressor (or vice versa). This of 


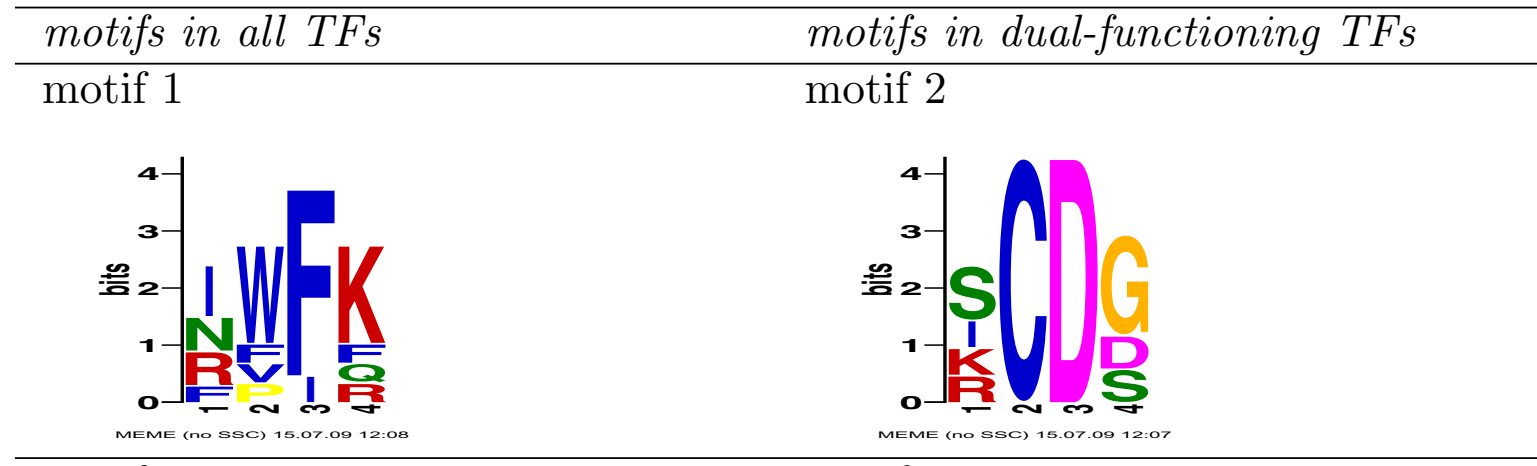

motif 3

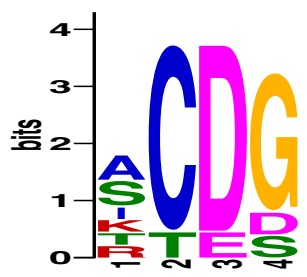

MEME (nO SSC) 15.070912 :08

motif 5

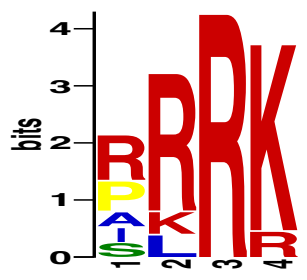

MEME (no SSC) 15.07.09 12:0

motif 7

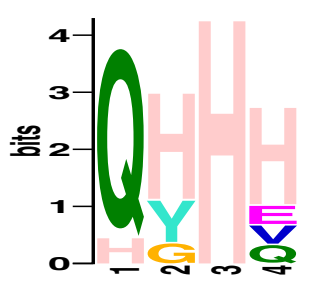

MEME (no SSC) 15.07.09 12:08

motif 9

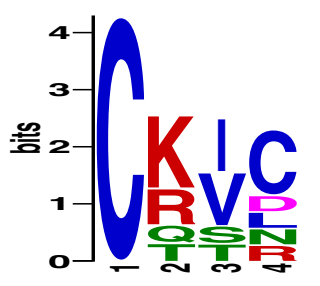

MEME (no SSC) 15.07.09 12:08 motif 4

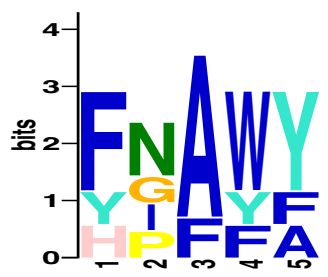

motif 6 (SUMO consensus)

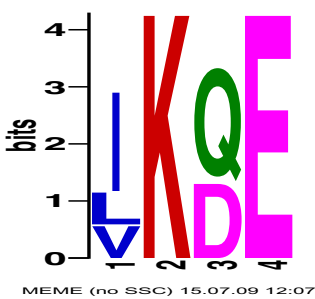

motif 8

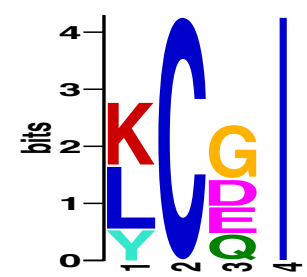

motif 10

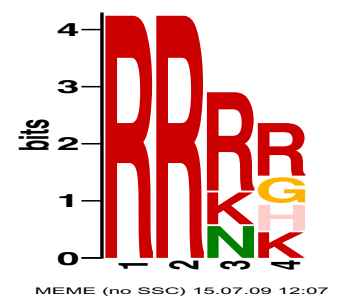

Figure 3 Motifs found in protein sequences according to MEME. Each panel shows the logo representation of a motif found in the protein sequence of all eight regulatory TFs (left column) or all TFs predicted to have dual function (right column). Motifs were found using MEME [22] in "OOPS" mode with a minimum sequence length of four and a maximum of six. 
Table 5: Role of TFs in comparison with presence of SUMOylation consensus motif and the role reported in the literature.

\begin{tabular}{|c|c|c|c|c|c|c|c|c|}
\hline & Bcd & Cad & $H b$ & TII & Gt & $K r$ & Kni & TorRE \\
\hline roles & $\mathbf{s}$ & + & $\mathbf{s}$ & - & $\mathbf{s}$ & $\mathbf{s}$ & - & $\mathbf{s}$ \\
\hline $\begin{array}{c}\text { Number of SUMO } \\
\text { sites }\end{array}$ & 1 & 0 & 2 & 0 & 1 & 2 & 0 & 1 \\
\hline Perkins et al. [29] & + & + & s & - & s & s & - & NA \\
\hline Schroeder et al. [9] & + & + & s & - & - & $(\mathrm{s})$ & - & + \\
\hline Rivera-Pomar et al. [35] & + & + & s & - & - & s & - & NA \\
\hline Sanchez et al. [36] & + & + & s & - & - & - & - & NA \\
\hline Jaeger et al. [37] & + & + & $s$ & - & $s$ & $s$ & $s$ & NA \\
\hline
\end{tabular}

The first row indicates the role we assign to the TFs, where "+" indicate activators, "-" indicate repressors, and "s" indicates a TF switching roles. The second row shows the number of sites found in the protein sequence of the TF that match the SUMO-consensus motif. The last rows summarizes the roles for the TFs previously reported in the literature.

course does not rule out the possible involvement of some other TF whose DNA-binding motif is not yet known; Nor does it address the possibility of an unknown non-DNA-binding TF being the mechanism required to explain the inability of the thermodynamic model to successfully fit the data. We hypothesize a SUMOylationpropelled mechanism for the TF role-switch in the different CRMs. Support for this idea is provided by the fact that SUMO consensus motifs are present in exactly the subset of TFs that our thermodynamic model-based study predict to be role-switchers. Our hypothesis is in line with prior evidence linking SUMOylation to the modulation of transcriptional activity of TFs [30-32]. This previous work has shown that the SUMOylation status of a TF affects which cofactors it can interact with, thus modulating its affect on transcription. Our hypothesis is also made more plausible by the fact that the SUMO homologue in Drosophila, Smt3, is uniformly distributed throughout the embryo $[17,33]$. In conclusion, our results suggest that current assumptions of a uniform, fixed regulatory mechanism for all developmental CRMs should be revised towards a model allowing for dual roles to improve prediction accuracy. Furthermore, our proposed mechanism of a SUMOylation-dependent switch represents a promising area for further research, and might be another step towards uncovering the biological mechanisms underlying the transcriptional regulation of the $D$. melanogaster developmental network. A full knowledge of this system will be one of the breakthroughs needed for a quantitative understanding of transcription and ultimately the development of accurate predictive models of gene expression from DNA sequences.

\section{Methods}

\subsection{Static, thermodynamic models of transcription}

We consider two thermodynamic models referred to as the Reinitz model [3] and the Segal model [5]. In a nutshell, both approaches model the transcription rate of a gene as a function of a vector of free model parameters, $\Theta$, and a vector of model inputs, $\mathbf{X}$. The model inputs comprise the set of regulatory TFs, the protein concentrations of these TFs and a set of log-odds (base two) scores describing the TF binding affinity along the controlling CRM. Latter is obtained by scanning the sequence of the CRM using a position weight matrix (PWM) representation of the TF binding preference and a PWM scanner, e.g. FIMO [22]. In addition, the Reinitz models requires a pre-specified role activator or repressor - for each of the TFs as input, while the Segal model optimizes the role during training. We represent the role of the $n$ TFs by a binary vector $\phi=\left\langle r_{1}, r_{2}\right.$, $\left.<, r_{\mathrm{n}}\right\rangle$, where $r_{i}=1$ indicates TF $i$ is an activator, and $r_{i}=-1 \mathrm{a}$ repressor, respectively. We refer to $\phi$ as a "configuration".

While the Segal model uses the log-odds binding scores at all positions in the CRM, the Reinitz model discretizes them by identifying individual (high-scoring) TFBSs when applying a PWM score threshold, $t$. We call a set of positions and log-odds scores "TFBS-map".

The set of free parameters, $\Theta$, of the Reinitz model is considerably smaller than that of the Segal model. Reinitz's set of parameters comprises a maximum association constant $(K)$, an "effectiveness" constant $(E)$ for each TF, the Gibbs free energy threshold $\left(G_{0}\right)$ of transcription and the maximal transcription rate $\left(R_{0}\right)$. This comes to $2 n+2$ parameters in $\Theta_{\text {Reinitz }}$, where $n$ is the number of TFs. In 
contrast, $\left|\Theta_{\text {Segal }}\right|=3 n+3|W|+|C|$, where $|W|$ is the overall sum of the length of each PWM for the TFs and $|C|$ is the number of CRMs. The three parameters per TF comprise the concentration scaling factor, which is comparable to $K$, and the expression contribution, which is comparable to $E$, while the third free parameter is the self-cooperativity value, which has no counterpart in the Reinitz model as synergy between TFs is not modeled there. The second term of the sum covers free parameters for the PWM, which are optimized for each TF during training. Finally, for every CRM, $c$, in the data set there is a free parameter describing its basal transcription rate, $G_{0}^{c}$.

\section{Data sets}

We use the D. melanogaster gap-gene data set [5]. The data set comprise spatial and temporal concentration patterns of eight TF proteins: Bicoid (Bcd), Caudal (Cad), Giant (Gt), Hunchback (Hb), Knirps (Kni), Krüppel (Kr) Tailless (Tll) and Torso-Response-Element (TorRE). The data set also contains estimates of the transcriptional response mediated by 44 CRMs, as measured by mRNA levels generated by lacZ-reporter constructs containing each of the CRMs. The protein and mRNA measurements are made at the same developmental time points at multiple points across entire $D$. melanogaster embryos. The data set also contains the DNA sequence for each of the 44 CRMs. We use a PWM describing the binding preferences of the TF and the motif scanning program FIMO [22] to predict discrete TFBSs within the regulatory regions. As described in Sec. 0.2, an important feature of the Segal model is to adjust the known PWMs. Altering the binding preference of each TFs allows the model to better describe the observed data but may, at the same time, introduce a bias towards the data set at hand. We use both the standard ("off-the-shelf") PWMs [7], and the "tweaked" PWMs to generate a TFBS-map. Since Segal et al. [5] used a uniform background frequency to calculate the log-odds score we also use a uni- form background for the tweaked PWMs, and a $D$. melanogaster specific background frequency, $F_{A}=F_{T}=$ $0.297 F C=F G=0.203$, when using the "off-the-shelf" PWMs. The Reinitz model normalizes the PWM scores for the TFBSs of TF $a$ in the data set by the maximal possible score, $S_{\text {max }}^{a}$, which is the sum of the largest log-odds score in every column of the PWM. We use a PWM threshold $t=9$ bits.

The Reinitz model allows for distance-dependent repression, where the effect of activators are reduced when they are within $d$ base pairs of an occupied repressor site. The Segal model does not have a distance-dependent repression mechanism. Instead, a repressor reduces the effect of all bound activators within the CRM. If the distance parameter in the Reinitz model is set to a number larger than the extent of the CRM, the two models should exhibit the same behavior with respect to the repressor function.

\subsection{Training on multiple CRMs and different configurations}

To measure how well each configuration can fit each CRM in the Segal single-time data, we train one Reinitz model for each possible configuration and each of the 44 developmental CRMs individually using the SA optimizer with geometric cooling schedule [23]. SA was allowed to optimize for 1000 iterations and the resulting $\mathrm{CC}$ is averaged over five independent repeats. This generates a $44 \times 256$ matrix with the average $\mathrm{CC}$ values of each configuration on each CRM.

By training a Reinitz model on each CRM individually, rather than training one model on all CRMs simultaneously we obtain an upper bound of how well the Reinitz model can fit each CRM. The small size of the training data set almost certainly causes the model to over-fit and, hence, the resulting $\mathrm{CC}$ values are likely overly optimistic. So, failure to produce good CC values is hence an even stronger indication for that the model is not able to fit the CRM with a given configuration of TF roles. To determine the roles for a TF from the CC matrix, we use the vote of three different methodologies, each focusing on a different aspect of the data, therefore avoiding the bias and isolating the signal picked up by the majority. These methodologies are called "Role-determining methods" and are described in the next section.

\subsection{Role-determining methods}

We employ three different role-determining approaches: the SMALLEST-OPTIMAL method, $\cup$; the Best-N method, $\beta$; and the SENSITIVITY method, $\Delta$. Each of these approaches is based on analyzing how well the Reinitz model fits the data for an individual CRM and configuration. 


\section{Smallest-optimal (set of configurations)}

The smallest optimal set of configurations is chosen such that each individual CRM is assigned to its optimal configuration, determined by the best individual training accuracy (CC). If a particular TF has no influence on the regulation of a CRM, that is if all the configurations-pairs with the TF in different roles have a similar CC--that is, if the difference in CC between the two methods, $\Delta$, is less than a constant, $\epsilon$--the role of the TF is set to "NA". We use $\epsilon=0.1$. Any role predictions other than "NA" are defined as "strong" in this method. While this method is guaranteed to identify the configurations with the absolute best accuracy, we expect the achieved $\mathrm{CC}$ values to be over-fitted, hence identifying the best performing CC might be noisy.

\section{Best-N}

The BEST-N method chooses the set of $n$ different configurations that jointly achieve the optimal performance on all CRMs. This greedy approach is computationally expensive since there are $\left(\begin{array}{c}256 \\ n\end{array}\right)$ combinations to test and hence, in this form, it is only feasible up to $n=4$. However, since we assume that a small set of different configurations is able to explain the regulation of the 44 CRMs, there may be no need for an exhaustive calculation for $n>4$. This approach always assigns a CRM to a configuration, we therefore receive "strong" role predictions for each TF in every CRMs. Similar to the SMALLEST-OPTIMAL method, the reliance on the accuracy measure might be a problem given that we assume them to be over-fitted.

\subsubsection{Sensitivity}

The SENSITIVITY method identifies a particular role for each TF in a CRM rather than the best (set of) configuration(s). The role $r$ is chosen for TF $t$ in CRM $c$ if it is "critical"--that is, if switching to it causes the largest increase in fitting accuracy. For a given CRM-TF pair, we compute the difference in performance between all pairs of configurations that differ only in the role of the TF, and assign the TF the role that causes the maximum increase in fitting accuracy. This is described in detail in the next paragraph. Let $\phi$ be a configuration specifying the roles of each of the 8 TFs. Let $A(\phi, t)$ be $\phi$ with the role of TF $t$ set to "activator", whereas $R(\phi, t)$ sets the role of $t$ to "repressor". Let $C C(c, \phi)$ be the correlation coefficient of the expression model fit to CRM $c$ using configuration $\phi$. Then, for CRM $c$, the maximum improvement in fit accuracy we can obtain when we switch $t$ from "repressor" to "activator" is given by

$$
\Delta C C_{c, t}=\max _{\phi}(C C(c, A(\phi, t))-C C(c, R(\phi, t)) .
$$

We assign the role of $t$ according to the sign of the maximum difference in accuracy, making no assignment if the difference is too small:

$$
r_{c, t}= \begin{cases}+1 & \text { if } \Delta C C_{c, t}>0.1 \\ -1 & \text { if } \Delta C C_{c, t}<-0.1 \\ \text { NA } & \text { otherwise }\end{cases}
$$

We define a role prediction as "strong" in this method if the absolute value of $\triangle C C>0.1$.

\subsubsection{Defining single- and dual-functioning TFs}

To identify if a TF has a single role for each CRM, we first combine for each method individually the evidence for all CRMs by counting how many times this particular TF was preferred as activator and repressor and calculating a fold-change over one or the other role. We translate this fold-change to mean the TF is a consistently an activator or repressor if more than $\frac{2}{3}$ of the CRMs favoured the TF as activator and repressor, respectively. If for less than $\frac{2}{3}$ of the CRMs the function agreed, We define the TF to switch roles. We then combine the evidence for single- or dual-function obtained for the role-determining methods individually by building a majority vote over the assigned functions.

\subsection{Training the model when dual-function is allowed}

To perform the training with dual-functioning TFs, we first use the SENSITIVITY method (described in Sec. 0.4.1) to determine the set of CRMs for which the TF was favoured in one or the other role by training a model on every CRM-configuration pair. CRMs where no role was determined by the SENSITIVITY method were assigned to configurations using the TF in its literature configuration. The TFBS-map is then adjusted such that only the appropriate version of the TF--activator or repressor--can bind to the TFBSs in the map according to what the SENSITIVITY method had determined for this CRM. Note, the protein concentrations remain the same for the TF irrespective of its function. We then train on all $44 \mathrm{CRMs}$ simultaneously as described in Sec. 0.3, except that now two free parameters describe the effectiveness of the TF as activator, $E_{\text {activator }}$, and repressor, $E_{\text {repressor }}$, respectively. 


\subsection{Enrichment analysis}

We perform motif enrichment analysis in order to discover if other site-specific TFs might be involved in the regulation of the developmental genes in D. melanogaster. The assumption is that if a TF is either directly involved in the regulation of the genes, or mediating roleswitching by one of the eight TFs, it will have more binding sites in the set of CRM sequences that require the TF to be an activator compared to the set of sequences that require it to be a repressor (or vice versa). We call TFs that are over-represented in one set of CRM sequences compared to the other "differentially" enriched.

We use CLOVER [19] to calculate the $p$-value for overor under-representation of TFBSs given a specific TF and a set of sequences compared with all intergenic regions in D. melanogaster. We collect two sequence sets, which were predicted to be subject to a different regulatory mechanism: set $A$ were $\mathrm{Hb}$ was predicted to be an activator and set $R$ where $\mathrm{Hb}$ was predicted o be a repressor, for $\mathrm{Kr}$ respectively. For each of these sequence sets we run CLOVER [19] to identify TFs whose TFBSs are enriched in one or the other set. We call a TF to be over-represented if the $p$-value is below 0.01 and under-represented if the $p$-value is above 0.99 . We report all TFs with known binding specificity taken from http://www.danielpollard.com/matrices.html and Slp1 from JASPAR [34] (76 in total) that are overrepresented in set $A$ or $R$. For those TFs to be called "differentially" enriched we require the difference in $p$-values between set $A$ and $R$ to be larger than 0.2 .

\section{Additional material}

Additional File 1 Supporting material for the article. The file contains Sections 1 to 6 referred to in the article.

\section{Authors' contributions}

$D C B$ researched and carried out the experimental work under the supervision of TLB. FAB designed the BEST-N method. The initial manuscript draft was written by $D C B$, and refined by FAB and TLB. All authors read and approved the final manuscript.

\section{Acknowledgements}

The authors wish to acknowledge John Reinitz and Eran Segal for helpful comments on the experimental set-up.

\section{Author Details}

Institute for Molecular Bioscience, The University of Queensland, Brisbane, Qld. 4072 Australia

Received: 16 November 2009 Accepted: 2 July 2010

Published: 2 July 2010

\section{References}

1. Ptashne M: Regulation of transcription: from lambda to eukaryotes. Trends Biochem Sci 2005, 30(6):275-279.

2. Reinitz J, Hou S, Sharp DH: Transcriptional Control in Drosophila. Complexus 2003, 1:54-64.

3. Janssens H, Hou S, Jaeger J, Kim AR, Myasnikova E, Sharp D, Reinitz J: Quantitative and predictive model of transcriptional control of the
Drosophila melanogaster even skipped gene. Nat Genet 2006, 38(10):1159-1165.

4. Zinzen RP, Senger K, Levine M, Papatsenko D: Computational models for neurogenic gene expression in the Drosophila embryo. Curr Biol 2006, 16(13):1358-1365.

5. Segal E, Raveh-Sadka T, Schroeder M, Unnerstall U, Gaul U: Predicting expression patterns from regulatory sequence includes Drosophila segmentation. Nature 2008, 451(7178):535-540.

6. Gertz J, Siggia ED, Cohen BA: Analysis of combinatorial cis-regulation in synthetic and genomic promoters. Nature 2008, 457:215-218.

7. Bauer DC, Bailey TL: Studying the functional conservation of cisregulatory modules and their transcriptional output. $B M C$ Bioinformatics 2008, 9:220.

8. Bauer DC, Bailey TL: Optimizing static thermodynamic models of transcriptional regulation. Bioinformatics 2009, 25(13):1640-1646.

9. Schroeder MD, Pearce M, Fak J, Fan H, Unnerstall U, Emberly E, Rajewsky N, Siggia ED, Gaul U: Transcriptional control in the segmentation gene network of Drosophila. PLoS Biol 2004, 2(9):E271.

10. Ma J: Crossing the line between activation and repression. Trends Genet 2005, 21:54-59.

11. Small S, Blair A, Levine M: Regulation of even-skipped stripe 2 in the Drosophila embryo. EMBO J 1992, 11(11):4047-4057.

12. Reinitz J, Kosman D, Vanario-Alonso CE, Sharp DH: Stripe forming architecture of the gap gene system. Dev Genet 1998, 23:11-27.

13. Papatsenko D, Levine MS: Dual regulation by the Hunchback gradient in the Drosophila embryo. Proc Natl Acad Sci USA 2008, 105(8):2901-2906.

14. Yu J, Pacifico S, Liu G, Finley RL: DrolD: the Drosophila Interactions Database, a comprehensive resource for annotated gene and protein interactions. BMC Genomics 2008, 9:461.

15. Makeev VJ, Lifanov AP, Nazina AG, Papatsenko DA: Distance preferences in the arrangement of binding motifs and hierarchical levels in organization of transcription regulatory information. Nucleic Acids Res 2003, 31(20):6016-6026

16. Müller S, Ledl A, Schmidt D: SUMO: a regulator of gene expression and genome integrity. Oncogene 2004, 23(11):1998-2008.

17. Talamillo A, Sánchez J, Barrio R: Functional analysis of the SUMOylation pathway in Drosophila. Biochem Soc Trans 2008, 36(Pt 5):868-873.

18. Makhnevych T, Sydorskyy Y, Xin X, Srikumar T, Vizeacoumar FJ, Jeram SM, Li Z, Bahr S, Andrews BJ, Boone C, Raught B: Global map of SUMO function revealed by protein-protein interaction and genetic networks. Mol Cell 2009, 33:124-135.

19. Frith MC, Fu Y, Yu L, Chen JF, Hansen U, Weng Z: Detection of functional DNA motifs via statistical over-representation. Nucleic Acids Res 2004, 32(4):1372-1381.

20. Gupta S, Stamatoyannopoulos JA, Bailey TL, Noble WS: Quantifying similarity between motifs. Genome Biol 2007, 8(2):R24.

21. Andrioli LP, Oberstein AL, Corado MSG, Yu D, Small S: Grouchodependent repression by sloppy-paired 1 differentially positions anterior pair-rule stripes in the Drosophila embryo. Dev Biol 2004 276(2):541-551.

22. Bailey TL, Boden M, Buske FA, Frith M, Grant CE, Clementi L, Ren J, Li WW, Noble WS: MEME Suite: tools for motif discovery and searching. Nucleic Acids Res 2009:W202-W208.

23. Bauer DC, Buske FA, Bailey TL, Bodén M: Predicting SuMOylation sites in developmental transcription factors of Drosophila melanogaster. Neurocomputing 2009 in press.

24. Valin A, Gill G: Regulation of the dual-function transcription factor Sp3 by SUMO. Biochem Soc Trans 2007, 35(Pt 6):1393-1396.

25. Yamamoto $H$, Ihara $M$, Matsuura $Y$, Kikuchi A: SUMOylation is involved in beta-catenin-dependent activation of Tcf-4. EMBO J 2003 22(9):2047-2059.

26. Hwang EJ, Lee JM, Jeong J, Park JH, Yang Y, Lim JS, Kim JH, Baek SH, Kim KI: SUMOylation of RORalpha potentiates transcriptional activation function. Biochem Biophys Res Commun 2009, 378(3):513-517.

27. del Arco PG, Koipally J, Georgopoulos K: Ikaros SUMOylation: switching out of repression. Mol Cell Biol 2005, 25(7):2688-2697.

28. Stielow B, Sapetschnig A, Wink C, Krüger I, Suske G: SUMO-modified Sp3 represses transcription by provoking local heterochromatic gene silencing. EMBO Rep 2008, 9(9):899-906.

29. Perkins TJ, Jaeger J, Reinitz J, Glass L: Reverse engineering the gap gene network of Drosophila melanogaster. PLoS Comput Biol 2006, 2(5):e51. 
30. Wang J, Feng XH, Schwartz RJ: SUMO-1 modification activated GATA4dependent cardiogenic gene activity. J Biol Chem 2004, 279(47):49091-49098.

31. Perdomo J, Verger A, Turner J, Crossley M: Role for SUMO modification in facilitating transcriptional repression by BKLF. Mol Cell Biol 2005, 25(4):1549-1559.

32. Wei F, Schöler HR, Atchison ML: Sumoylation of Oct4 enhances its stability, DNA binding, and transactivation. J Biol Chem 2007, 282(29):21551-21560.

33. Lehembre F, Badenhorst P, Müller S, Travers A, Schweisguth F, Dejean A: Covalent modification of the transcriptional repressor tramtrack by the ubiquitin-related protein Smt3 in Drosophila flies. Mol Cell Biol 2000 20(3):1072-1082.

34. Portales-Casamar E, Thongjuea S, Kwon AT, Arenillas D, Zhao X, Valen E, Yusuf D, Lenhard B, Wasserman WW, Sandelin A: JASPAR 2010: the greatly expanded open-access database of transcription factor binding profiles. Nucleic Acids Res 2010:D105-D110.

35. Rivera-Pomar R, J228; ckle H: From gradients to stripes in Drosophila embryogenesis: filling in the gaps. Trends Genet 1996, 12(11):478-483.

36. Sánchez L, Thieffry D: A logical analysis of the Drosophila gap-gene system. J Theor Biol 2001, 211(2):115-141.

37. Jaeger J, Blagov M, Kosman D, Kozlov KN, Manu, Myasnikova E, Surkova S, Vanario-Alonso CE, Samsonova M, Sharp DH, Reinitz J: Dynamical analysis of regulatory interactions in the gap gene system of Drosophila melanogaster. Genetics 2004, 167(4):1721-1737.

doi: 10.1186/1471-2105-11-366

Cite this article as: Bauer et al, Dual-functioning transcription factors in the developmental gene network of Drosophila melanogaster BMC Bioinformatics 2010, 11:366

Submit your next manuscript to BioMed Central and take full advantage of:

- Convenient online submission

- Thorough peer review

- No space constraints or color figure charges

- Immediate publication on acceptance

- Inclusion in PubMed, CAS, Scopus and Google Scholar

- Research which is freely available for redistribution 